Network Working Group

D. Wing

Request for Comments: 2530

Cisco Systems

Category: Standards Track

March 1999

\title{
Indicating Supported Media Features Using \\ Extensions to DSN and MDN
}

Status of this Memo

This document specifies an Internet standards track protocol for the Internet community, and requests discussion and suggestions for improvements. Please refer to the current edition of the "Internet Official Protocol standards" (STD 1) for the standardization state and status of this protocol. Distribution of this memo is unlimited.

Copyright Notice

Copyright (C) The Internet Society (1999). All Rights Reserved.

1. Abstract

There is a need in Internet mail and Internet fax for a recipient to indicate the media features it supports so that messages can be generated by senders without exceeding the recipient's abilities.

This memo describes a format for generating Message Disposition Notifications [RFC2298] and Delivery Status Notifications [RFC1894] which contain such information. This information can be used by senders to avoid exceeding the recipient's capabilities when sending subsequent messages.

2. Introduction

The extensions described in this document can be used in Message Disposition Notifications [RFC2298] or Delivery Status Notifications [RFC1894], as appropriate for the implementation.

Note that both DSNs and MDNs have drawbacks: DSNs are not available between all senders and receivers, and MDNs require the receiver to disclose message disposition information (or, if using the "denied" disposition-type, the time the disposition notification was generated).

The key words "MUST", "MUST NOT", "REQUIRED", "SHALL", "SHALL NOT", "SHOULD", "SHOULD NOT", "RECOMMENDED", "MAY", and "OPTIONAL" in this document are to be interpreted as described in [RFC2119]. 
3. Extensions for use by DSN and MDN

The following extension is available to both DSN [RFC1894] and MDN [RFC2298] messages.

For a DSN message, the following per-recipient fields are defined (section 2.3 of [RFC1894]). For an MDN message, the following extension fields are defined (section 3.1 of [RFC2298]). Using the language of [RFC2234]:

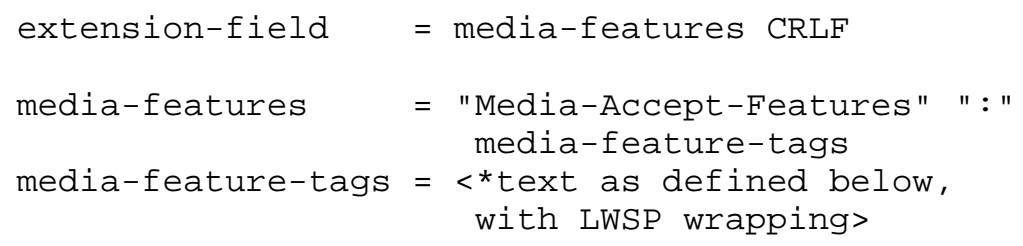

The <media-feature-tags> are defined in separate schema documents which MUST utilize the language described in [SYNTAX]. The schema MUST be registered following the registration requirements of [RFC2506].

\subsection{Examples}

The following examples assume there is a schema document which defines the tags shown.

3.1.1. Paper-size and Color

Assuming there is a schema document which describes the tags papersize and color, the following example is valid:

Media-Accept-Features: (\& (paper-size=a4) (color=binary) )

3.1.2. UA-Media, Paper-size, and Color

Assuming there is a schema document which describes the tags papersize, color, and grey:

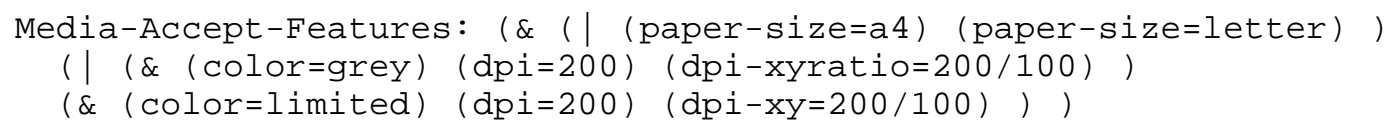

4. MTA Implmentation Recommendation

If the recipient's MTA determines that a message cannot be processed, the recipient's MTA is strongly encouraged to reject the message with a status code of 5.6.1 [RFC1893]. This status code may be returned 
in response to the end-of-mail-data indicator if the MTA supports reporting of enhanced error codes [RFC2034], or after message reception by generating a delivery failure DSN ("bounce").

5. Security Considerations

Inaccurate media feature information could cause a denial of service, causing subsequent messages to be sent which the recipient is unable to process.

The media feature information could be inaccurate due to a malicious attack (spoofed DSN or MDN) or misconfiguration.

6. Acknowledgments

The author thanks the members of the Internet Fax working group for assistance with this document, and especially Larry Masinter, Graham Klyne, and Ned Freed.

\section{References}

[RFC2506] Holtman, K., Mutz, A. and T. Hardie, "Media Feature Tag Registration Procedure", BCP 31, RFC 2506, March 1999.

[RFC1894] Moore, K. and G. Vaudreuil, "An Extensible Message Format for Delivery Status Notifications", RFC 1894, January 1996.

[RFC2034] Freed, N., "SMTP Service Extension for Returning Enhanced Error Codes", RFC 2034, October 1996.

[RFC2119] Bradner, S., "Key words for use in RFCs to Indicate Requirement Levels", BCP 14, RFC 2119, March 1997.

[RFC2234] Crocker, D. and P. Overell, "Augmented BNF for Syntax Specifications: ABNF", RFC 2234, November 1997.

[RFC2298] Fajman, R., "An Extensible Message Format for Message Disposition Notifications", RFC 2298, March 1998.

[SYNTAX] Klyne, G., "A Syntax for Describing Media Feature Sets", RFC 2533, March 1999. 
8. Author's Address

Dan Wing

Cisco Systems, Inc.

101 Cooper Street

Santa Cruz, CA 95060 USA

Phone: +1 8314575200

Fax: $\quad+18314575208$

EMail: dwing@cisco.com 
9. Full Copyright statement

Copyright (C) The Internet Society (1999). All Rights Reserved.

This document and translations of it may be copied and furnished to others, and derivative works that comment on or otherwise explain it or assist in its implementation may be prepared, copied, published and distributed, in whole or in part, without restriction of any kind, provided that the above copyright notice and this paragraph are included on all such copies and derivative works. However, this document itself may not be modified in any way, such as by removing the copyright notice or references to the Internet society or other Internet organizations, except as needed for the purpose of developing Internet standards in which case the procedures for copyrights defined in the Internet standards process must be followed, or as required to translate it into languages other than English.

The limited permissions granted above are perpetual and will not be revoked by the Internet society or its successors or assigns.

This document and the information contained herein is provided on an "AS IS" basis and THE INTERNET SOCIETY AND THE INTERNET ENGINEERING TASK FORCE DISCLAIMS ALL WARRANTIES, EXPRESS OR IMPLIED, INCLUDING BUT NOT LIMITED TO ANY WARRANTY THAT THE USE OF THE INFORMATION HEREIN WILL NOT INERINGE ANY RIGHTS OR ANY IMPLIED WARRANTIES OF MERCHANTABILITY OR FITNESS FOR A PARTICULAR PURPOSE. 